\title{
ESTUDO COMPARATIVO ENTRE OS EFEITOS DA MASSAGEM CLÁSSICA RELAXANTE, MÉTODO IMEDIATT E MASSAGEM YBÁ PARA GESTANTES A PARTIR DO TERCEIRO TRIMESTRE
}

Daphne Bastos, Isabella Anázia, Liandra Souza, Ligia Faria, Milena Mello, Thayssa

Gatt e André Leonardo da Silva Nessi

Bacharelado em Estética, Escola de Ciências da Saúde - UAM - SP

Email: alnessi@anhembi.br

Acesso DOI: http://dx.doi.org/10.34059/ciejop.2019v27i1-12

\section{RESUMO}

Introdução: A gestação é o período de desenvolvimento de um novo ser. Nessa fase da vida da mulher ocorrem muitas mudanças relacionadas aos hormônios. Entre essas mudanças estão inchaço, aumento da frequência de miç̧ão, enjoos e náuseas, ganhos de massa corporal, tensões musculares e desconfortos. Para aliviar esses desconfortos há no mercado diferentes técnicas de massagem. A massagem manual para gestantes, o método Imediatt e a massagem Ybá tratam-se de recursos manuais que proporcionam uma série de benefícios à gestante, cada qual com suas particularidades. A massagem manual proporciona alívio de tensões musculares, diminui a ansiedade e a dor, além de produzir benefícios emocionais. A massagem Imediatt para gestantes promove a melhora de edema e relaxamento da gestante. A massagem Ybá relaxa a mãe e acalma o bebê por meio do toque, aromaterapia, musicoterapia e cromoterapia através da seda. Objetivo: comparar os efeitos da massagem clássica relaxante, método Imediatt e massagem Ybá para gestantes a partir do terceiro trimestre. Metodologia: Este estudo comparativo e qualitativo, consiste em aplicar as três diferentes técnicas de massagem com a coleta de dados (pressão arterial, frequência cardíaca e perimetria), antes e após o procedimento, para mensurar os reais benefícios de cada técnica e compará-las. Resultados: Ao final das massagens observou-se aumento da frequência cardíaca e da pressão arterial, resultantes do débito cardíaco no período gestacional, onde é aumentado (30 a 50\%) atingindo seu valor máximo entre a 20 (vigésima) e 40 (quadragésima) semana, o que torna mais sensível as alterações posturais (devido ao fato do útero em crescimento, comprimi a veia cava inferior, diminuindo o retorno venoso). Conclusão: Observou-se após o término das massagens que os métodos Massagem Clássica Relaxante, Método Ybá e Método Imediatt, apresentaram bons resultados na avaliação para o sono e alívio de dores nas costas. Na diminuição de edema por meio da perimetria o Método Imediatt teve melhores resultdos. Em questão do relaxamento, as gestantes relataram melhor sensação de leveza e conexão com o bebê em especial para massagem Ybá. Concluiu-se que a cliente gestante deve receber os tratamentos de massagem nesse período para promover bem-estar, relaxamento, melhorar edemas e ter mais conexão com a vida que está gerando.

Palavras-chave: massagem; fisiologia; gestantes.

SUMMARY

Introduction: Gestation is the period of development of a new being. At this stage of a woman's life many hormone-related changes occur. Among these changes are swelling, increased frequency of urination, nausea and sickness, body mass gains, muscle tensions and discomforts. To relieve these discomforts there are different 
massage techniques on the market. The manual massage for pregnant women, the Imediatt method and the Ybá massage are manual resources that provide a series of benefits to the pregnant woman, each one with its peculiarities. Manual massage provides relief from muscle tensions, decreases anxiety and pain, and produces emotional benefits. The Imediatt massage for pregnant women promotes the improvement of edema and relaxation of the pregnant woman. Ybá massage relaxes the mother and calms the baby through touch, aromatherapy, music therapy and chromotherapy through silk. Objective: To compare the effects of classic relaxing massage, Imediatt method and Ybá massage for pregnant women from the third trimester. Methodology: This comparative and qualitative study consists of applying the three different techniques of massage with data collection (blood pressure, heart rate and perimetry), before and after the procedure, to measure the real benefits of each technique and to compare them . Results: At the end of the massages, heart rate and blood pressure increased, resulting from cardiac output in the gestational period, where it increased (30 to 50\%) reaching a maximum value between 20 (twentieth) and 40 (fortieth) week, which makes postural changes more sensitive (due to the fact that the growing uterus compresses the inferior vena cava, decreasing venous return). Conclusion: It was observed after the end of the massages that the methods Relaxing Classical Massage, Ybá Method and Imediatt Method, presented good results in the evaluation for sleep and relief of back pain. In decreasing edema through perimetry the Imediatt Method had better results. In terms of relaxation, the pregnant women reported a better sensation of lightness and connection with the baby, especially for Ybá massage. It was concluded that the pregnant client should receive the massage treatments in this period to promote wellbeing, relaxation, improve edema and have more connection with the life that is generating.

Keywords: massage; physiology; pregnant women.

\section{INTRODUÇÃO}

O período gestacional é uma condição de desenvolvimento para formação de um novo ser. Deve ser adotado um estilo de vida mais saudável como, por exemplo, alimentação equilibrada, prática de exercícios físicos, sono e repouso e boas relações familiares para que ocorra um bom desenvolvimento do bebê. Tratase de uma fase delicada e, para isso, são necessários cuidados extremos.

A gestação representa um período muito importante de transição na vida de uma mãe. Apresenta muitas mudanças em seu corpo e emocional, causando algumas sensações e desconfortos como a fadiga, dores lombares, insônia, inchaço, ansiedade, estresse, prisão de ventre, aumento da frequência de micção, enjoos, ganho de massa corporal, tensões musculares e desconforto causado pelo volume abdominal. 
A prática da massagem é definida por um conjunto de manobras metódicas que o terapeuta aplica sobre o corpo com objetivos reabilitadores, psicológicos, preventivos, higiênicos e terapêuticos (Mansilla, 2001).

A massagem deve ser realizada na gestante saudável e somente com autorização médica. A gestante deve ser posicionada em decúbito lateral ou ventral com o auxílio de uma maca específica para gestantes.

Entre os benefícios estão: diminuição de edemas, relaxamento, prevenção de hidrolipodistrofia ginóide, melhora da oxigenação e, consequentemente, nutrição do tecido, melhora da autoestima, prevenção de telangiectasias e alívio de tensões musculares, muito comuns nessa fase.

Neste texto, será revisado e comparado os efeitos que as massagens podem causar, melhorando e aliviando os desconfortos durante a gestação e podendo evitar algumas complicações pós-parto, como a depressão.

O objetivo comparar os efeitos da massagem clássica relaxante, método Imediatt e massagem Ybá para gestantes a partir do terceiro trimestre.

No período gestacional, a mulher revive suas experiências passadas, além de reajustar seu relacionamento conjugal, sua situação socioeconômica e suas atividades profissionais (Piccinini et al, 2008).

É dividida em três trimestres, sendo calculada em torno de quarenta semanas ou duzentos e oitenta e nove dias ou nove meses. Ocorrem transformações em todo o corpo da gestante para que ela possa abrigar seu bebê, alimentá-lo e permitir seu desenvolvimento até o nascimento (Fonseca et al, 2009). Entre essas transformações, citam-se alterações como em sua forma física, o surgimento de estrias na pele e alterações na alimentação. (Mann et al, 2010).

O estrogênio também é responsável por uma maior degradação de tecido adiposo nas mamas, fazendo com que elas cresçam, aumentando o número de célula glandular e tamanho dos ductos. A progesterona, praticamente, não exerce influência sobre os atributos sexuais femininos, mas sim, sobre o preparo do útero, para receber o óvulo fertilizado, e da mama, para a secreção do leite.

Durante a gravidez, a progesterona disponibiliza nutrientes para o feto, que ficam armazenados no endométrio. As mamas também recebem influência da progesterona, promovendo nutrientes nas células glandulares. Muitas vezes, a gestante pode passar por mudanças físicas e fisiológicas, verificando-se sintomas 
comuns no primeiro trimestre, que são o aumento da irritabilidade, cansaço, mudanças de apetite, prazer diminuído e distúrbios do sono (Baptista et al,2006).

No segundo trimestre, os distúrbios do sono são elevados, as alterações relacionadas ao sono podem ser originadas pelas mudanças fisiológicas na gravidez, como por exemplo, desconforto no posicionamento durante o sono, síndrome das pernas inquietas e pesadelos, tem menor duração do sono, desadormecer noturno, dificuldade de dormir, menor proveito do sono, fazendo com que as gestantes reclamem da má qualidade de sono.

A gestante apresenta o aumento de salivação que pode ser comprometido pelas mudanças hormonais e metabólicas. Durante as sensações bucais verificadas em gestantes, Pires (2008) destacou dois parâmetros salivar, como a xerostomia e a parageusia. A xerostomia é definida como a sensação de estar com a boca seca e tem redução do fluxo salivar, no entanto, podem ocorrer complicações orais que prejudicam a qualidade de vida da mulher. É importante saber que a xerostomia não reflete a uma redução das glândulas salivares, mas pode estar associada a uma desidratação. A parageusia também tem a redução do fluxo salivar e a sensação da boca seca, a mulher sente um sabor desagradável, principalmente o gosto amargo. Outro sintoma frequente, no segundo semestre, é o aumento da micção, justificado pelo aumento da taxa de filtração glomerular e o fluxo plasmático renal que contribuem para um aumento da excreção urinária de glicose, aminoácidos, proteínas e vitaminas. Como a função renal é muito sensível a postura durante à gravidez, regularmente aumentando a função na posição supina e diminuindo na posição vertical, quando tenta dormir pode sentir maior vontade de urinar(Moreira et.al,2007).

De acordo com Polden e Mantle (2005) começam a aparecer desejos, quer sejam por ordem orgânica ou ordem psicológica. Para Baracho (2007), deve realizar uma dieta, exercícios físicos, apresentar orientações posturais e orientar o uso de meias elásticas para aliviar a sintomatologia. Na dieta, temos três principais fontes, como o ácido fólico, ferro e cálcio, que não podem faltar na alimentação da mãe. $O$ primeiro é o ácido fólico, uma vitamina do complexo $B$, suas fontes alimentares são: folhas verde-escuras, ervilhas, feijões, laranja, germe de trigo, nozes, pão integral, ricota e iogurte; O segundo é o ferro e, como a anemia é muito comum na gravidez, é importantes ter uma alimentação rica em ferro, cujas fontes alimentares são: carnes, feijões e folhas verde-escuras. E, para melhorar o aproveitamento de ferro Fórum Internacional de Qualidade de Vida e Saúde - Curitiba, 15 de junho de 2019 
da refeição, é aconselhável sempre comer uma fruta rica em vitamina $\mathrm{C}$. O terceiro é o cálcio, um mineral importante para a formação dos ossos e dos dentes do bebê e suas fontes alimentares são: iogurte, leite e queijo.

A atividade física durante a gravidez é importante para manter o bem-estar e a disposição física e existem alguns fatores importantes. A má circulação e a compressão do útero sobre as veias aumentam edemas nos MMIl, pode também apresentar pré-eclampsia, definido por hipertensão arterial, edema corpóreo e aparecimento de albumina na urina (Baptista et.al,2006).

Segundo Beuttenmüller (2005), devido ao nervosismo em seu terceiro trimestre, nota-se o estado emocional alterado, ansiedade e cansaço pelo peso. A barriga gestacional em seu $9^{\circ}$ mês pode apresentar até 1 metro de circunferência, 0 útero pode alterar até 30 vezes mais que seu tamanho real, há também contrações com mais frequência e rapidez. Devido a descida do útero, a barriga se encontra mais baixa, com isso, pode-se proporcionar uma respiração melhor, entretanto, os indícios de parto podem aparecer (Fonseca et al,2009).

\section{Massagem manual na gestação}

A massagem é o ato de tocar no tecido, gerando um efeito terapêutico, e foi criada de forma empírica em Roma, Grécia, Índia e China. Cada lugar, com sua cultura, aplicavam-se a massagem de um jeito, sendo uma terapia não medicamentosa de promoção ao bem-estar e saúde. "Sendo tanto uma arte quanto uma ciência, sua evolução continuará enquanto continuar sendo explorada e pesquisada por estudantes e profissionais" (Santos,2015).

A massagem é considerada, há muito tempo, como um meio natural e instintivo de aliviar dores e desconforto porque, além de reduzir o estresse e a ansiedade, promove relaxamento e diminuição da fadiga muscular, possui ação sedativa e analgésica, aumenta a consciência corporal, produz benefícios emocionais e equilíbrio entre os sistemas simpático e parassimpático. Trata-se de uma técnica que, na gestação, diminui a ansiedade e a dor, conduzindo a parturiente de maneira satisfatória para o trabalho de parto (Bavaresco et.al,2011). Durante a gestação, devem ser utilizados produtos seguros tanto para a gestante, quanto para o bebê. Deve-se tomar mais cuidado por conta de alguns ativos que podem ter efeito abortivo, causarem más formações, alergias e alterações hormonais. Normalmente, 
nas massagens em gestantes, são utilizados óleos vegetais com óleo essencial (Urasaki,2010).

As contraindicações para a massagem na gestante são: anormalidades da placenta, anormalidades do útero, distúrbios do suprimento de sangue para o feto, o que aumenta a pressão arterial, múltiplos fetos, sangramento e risco de aborto, doença da pele, uso de heparina, inflamação aguda e flebite/tromboflebite. Nos três primeiros meses da gestação ocorrem grandes mudanças hormonais e fisiológicas (Montenegro e Rezende, 2010). A massagem nesse período não afeta o feto e não perturba os processos naturais, porém, por se tratar de uma fase muito delicada e importante para a gestante, devem ser evitadas quaisquer complicações. A massagem nesta fase em áreas abdominais e enquanto houver enjoos matinais ou vômitos também é contraindicada (Cassar, 2001).

A massagem no período gestacional conecta a mãe com o bebê e melhora os desconfortos dessa fase com movimentos leves e cuidadosos, como:

- Amassamento: melhora dores musculares, relaxando a gestante;

- Deslizamento: diminui tensões musculares e promove melhor consciência corporal;

- Fricção: alivia dores locais e

- Compressões drenantes: melhoram o retorno venoso e o edema.

As gestantes, normalmente, sentem muita dor na região lombar. É indicado que, nessa região, seja dada maior atenção com movimentos que relaxem e diminuam a tensão muscular. (Mata, Shimo,2017)

Segundo Sallet (2003), é recomendado o decúbito lateral à esquerda para a massagem em gestantes porque não ocorrerão interferências no retorno venoso, na perfusão uterina e na oxigenação fetoplacentária. $O$ abdome deve estar apoiado em almofadas para diminuir a instabilidade corporal. Trata-se de uma posição que elimina a pressão sobre a veia cava superior e aorta descendente, promovendo o maior conforto físico (Silva e Mejia,2014).

\section{MASSAGEM YBÁ}

É uma técnica desenvolvida pela esteticista e cosmetóloga Suellen Campanhola em sua própria clínica com pesquisas e estudos, com apoio dos obstetras Dr. Domingos e Dra. Erica Mantelli. 
A massagem utiliza da musicoterapia, cromoterapia (com o uso das sedas), aromaterapia e toque para beneficiar a gestante por meio do relaxamento, conexão com a vida intrauterina, melhora da circulação, estresse, insônia e tristeza. Dra. Erica destaca que o bebê também sente todas as sensações da mamãe. É um momento único que convida essa ligação muito importante para o desenvolvimento de um bom equilíbrio emocional.

Segundo MANTELLI, os adultos e adolescentes têm grande tendência a ter problemas psicológicos como a depressão e ansiedade, e para auxiliar na prevenção dessas patologias, pode-se utilizar dos benefícios da musicoterapia para melhor formação física e emocional do bebê. Durante a massagem Ybá utiliza-se uma música desenvolvida especialmente para o momento dessa massagem. A gestação mexe muito com as emoções da gestante, assim refletindo os efeitos do cortisol para o bebê, fazendo-se necessário o uso da música, para auxílio no relaxamento do corpo da mãe, acalmando o bebê.

Utiliza-se a aromaterapia para relaxar a gestante com óleo essencial de lavanda ou camomila (adequados para o período gestacional), respeitando o sensorial olfativo da mãe. (Mantelli, 2019)

As sedas utilizadas são em tons de azul (que trabalham emoções e sentimentos) para promover os efeitos da cromoterapia: sensação de tranquilidade, devoção, calma, verdade, sinceridade, meditação e poder do plano mental. (Mantelli, 2019)

A massagem só pode ser aplicada com a autorização do médico que realiza o acompanhamento da gestação. (Mantelli, 2019)

Têm duração de 30 a 35 minutos. Desperta sensação de acolhimento e aconchego, com movimentos rítmicos de vai e vem. Deve-se utilizar ou óleo vegetal ou orgânico.

Inicia-se em decúbito dorsal ou lateral (o que for mais confortável para a gestante), para as manobras em face, região peitoral, ombros, cabeça e membros superiores e inferiores. Para realizar as manobras em região cervical e lombar e posterior de coxa, a gestante é posicionada em decúbito lateral ou ventral (com a utilização de uma maca própria para gestantes). E é finalizada com a gestante sentada, massageando as costas e ventre levemente com a seda. Serve-se um chá de camomila ou lavanda para despertar o sentido do paladar e relaxá-la. (Mantelli, 2019) 


\section{MÉTODO IMEDIATT PARA GESTANTES}

É um método desenvolvido pela esteticista e cosmetóloga Ana Carolina Parreira. Trata-se de uma técnica de massagem desenvolvida para combater edemas, eliminar toxinas, reposicionar a musculatura e promover o relaxamento. É realizada de forma específica e adaptada. Antes de iniciar-se a Massagem Imediatt, deve-se avaliar a presença de edema, se o teste de cacifo for positivo, é realizada a drenagem linfática de membros superiores e inferiores com manobras de drenagem linfática do Método Leduc de acordo com a necessidade da cliente. Esse método promove bem-estar e relaxamento tanto para a futura mãe quanto para o bebê, diminuindo dores e tensões do período.

Uma pesquisa realizada em 2012 por Coutinho concluiu que num grupo de gestantes entrevistadas $61,3 \%$ incomodam-se com as alterações ocorridas no período gestacional, entre essas alterações destacam-se cansaço nas pernas, estrias, retenção de líquidos, manchas e ressecamento da pele. O estudo revela que o profissional de estética pode atuar de forma significativa e com resultados comprovados em até $80 \%$ dos problemas relatados pelas gestantes.

Todos os métodos de massagem devem ser precedidos por uma avaliação clínica do cliente. Numa boa avaliação é fundamental que o profissional compreenda o desejo e as expectativas do cliente para que possa elaborar o melhor protocolo e periodicidade. Para gestantes a autorização médica trata-se de um elemento obrigatório e pode ser anexado na ficha de anamnese. Devem-se respeitar todas as orientações médicas, portanto, se o obstetra autorizar a massagem somente em membros inferiores o profissional não deverá desrespeitar esta orientação.

A gestante deve ser acomodada de maneira confortável durante todo o atendimento. Os membros inferiores e a cabeça da gestante devem estar elevados. $\mathrm{O}$ ambiente deve estar numa temperatura agradável à gestante e o profissional deve demonstrar empatia, cuidado e atenção aos detalhes.

A drenagem linfática drena os líquidos excedentes que banham as células, desta forma, mantendo o equilíbrio hídrico dos espaços intersticiais. Dois processos distintos auxiliam na evacuação desses líquidos intersticiais. O primeiro processo é a captação: realizada pela rede de capilares linfáticos, é resultado do aumento local da pressão tissular. O segundo processo é a evacuação dos elementos recaptados pelos capilares. 
O método Leduc é reconhecido mundialmente, atua no sistema linfático, acelerando o processo de diminuição do edema. Nesta técnica são utilizados movimentos suaves, lentos e relaxantes proporcionando benefícios a estética, saúde e bem-estar. Entre as principais indicações para o método estão tratamento de edemas, gestantes e pós-operatório. As contraindicações são hipertensão descompensada, insuficiência renal, trombose venosa profunda, infecções de pele e erupções cutâneas, processos infecciosos e quando não há liberação médica. As manobras mais utilizadas do método são: manobra de Chamada (promove uma aspiração dos vasos linfáticos, responsável por aspirar e impulsionar a linfa dentro dos capilares) e a manobra de Reabsorção (promove aumento da pressão tissular e evacuação).

Não existe uma periodicidade específica e nenhum número preestabelecido de vezes em que cada manobra deva ser executada. A capacitação deste método permite ao profissional monitorar constantemente a resposta dos tecidos e ao mesmo tempo, as informações fornecidas pelo cliente.

\section{METODOLOGIA}

A pesquisa é comparativa e quantitativa. Consiste em aplicar a técnica de massagem clássica para gestantes, na primeira sessão, a técnica do método Imediatt na segunda sessão e a massagem Ybá, na terceira sessão, num grupo de 2 gestantes, com período gestacional compreendido entre a $25^{\mathrm{a}}$ e $42^{\mathrm{a}}$ semana. $O$ grupo deve ser constituído por gestantes que tenham autorização médica para receber as massagens.

A pesquisa que propomos será realizada em salas apropriadas, localizadas no SPA da Universidade Anhembi Morumbi, campus Centro-Mooca, São Paulo/SP.

Para coleta de dados, será aferida a PA (Pressão Arterial) de cada integrante do grupo, com o medidor de pulso da marca Omronno modelo HEM 6123. Em seguida, com a gestante em repouso, por 5 minutos, será medida a frequência cardíaca com o aparelho Power Lab e realizada a perimetria, com fita métrica, dos membros superiores e inferiores. Após, a gestante será recepcionada para 0 procedimento.

Os mesmos dados coletados no início de cada sessão, serão coletados novamente ao final para que os resultados possam ser comparados e analisados. 


\section{RESULTADOS}

Nas voluntárias quanto a perimetria, nos membros superiores MMSS e abdome, não ocorreram alterações significativas, mas em membros inferiores MMII, no método Imediatt, ocorreram diminuição de 61 para 58,5 na região da coxa, e de 37 para 35,5 na região do tornozelo. Um percentual médio de $4 \%$ na diminuição de edema localizado, nas voluntárias. Na Frequência Cardíaca ocorreu uma diminuição maior no método Ybá, 10\% na média das voluntárias. Nos três métodos, foram citadas pelas voluntárias: "Relaxada"; "Até dormi"; "Muito boa, o bebê ficou se mexendo"; "Muito boa"; "Relaxada"; "Sono; sinto leve e relaxada." "Muito gostosa, relaxei muito"; "Muito boa"; "Melhorou a dor na perna".

Ao final das massagens observou-se aumento da frequência cardíaca e da pressão arterial, resultantes do débito cardíaco no período gestacional, onde é aumentado (30 a 50\%) atingindo seu valor máximo entre a 20 (vigésima) e 40 (quadragésima) semana, o que torna mais sensível as alterações posturais (devido ao fato do útero em crescimento, comprimi a veia cava inferior, diminuindo o retorno venoso). No começo da gestação, a ejeção do sangue aumenta até $30 \%$, e se torna responsável pelo incremento do débito cardíaco. Com o passar dos meses, essa frequência vai aumentando, até que no final poderá apresentar 15 batimentos por minuto acima dos valores do início da gravidez (PICON, 2005).

\section{CONSIDERAÇÕES FINAIS}

Observou-se após o término das massagens que os métodos Massagem Clássica Relaxante, Método Ybá e Método Imediatt, apresentaram bons resultados na avaliação para o sono e alívio de dores nas costas. Na diminuição de edema por meio da perimetria o Método Imediatt teve melhores resultados. Em questão do relaxamento, as gestantes relataram melhor sensação de leveza e conexão com o bebê em especial para massagem Ybá. Concluiu-se que a cliente gestante deve receber os tratamentos de massagem nesse período para promover bem-estar, relaxamento, melhorar edemas e ter mais conexão com a vida que está gerando.

\section{REFERÊNCIAS}


BAPTISTA, Makilim. Associação entre suporte social, depressão, ansiedade em gestantes. Revista de Psicologia da Vetor Editora, Campinas, 2006. Disponível em: http://pepsic.bvsalud.org/scielo.php?pid=S1676-

73142006000100006\&script=sci_abstract\&tIng=en

BAVARESCO, Gabriela. O fisioterapeuta como profissional de suporte à parturiente. Ciência e Saúde Coletiva, Florianópolis, 2011. Disponível em:<http://www.scielo.br/scielo.php?script=sci_arttext\&pid=S1413-Acesso em 28 ago. 2018.

CASSAR, Mario. Manual de Massagem Terapêutica. In: CASSAR, Mario. Manual de Massagem Terapêutica. [S. I.]: Manole, 2001.

FONSECA, Floripes et al. Estudo comparativo entre a drenagem linfática manual e a atividade física em mulheres no terceiro trimestre de gestação. Fisioterapia Ser, Fortaleza, 2009. Disponível em: https://s3.amazonaws.com/academia.edu.documents/30985738/80feda297f20c391b b289271315079f2.pdf?AWSAccessKeyld=AKIAIWOWYYGZ2Y53UL3A\&Expires $=15$ 53832100\&Signature=q2h6wY4OKA\%2FTWGFcQGKryjEvowM\%3D\&responsecontent-

disposition=inline\%3B\%20filename\%3DTrauma_raquimedular_perfil_epidemiologic.p df\#page=13. Acesso em: 4 out. 2018.

FONSECA, Marcia et al. Prevalência do uso de medicamentos na gravidez: uma abordagem farmacoepidemiológica. Revista de Saúde Pública, Campinas, 2002. Disponível em: http://www.scielo.br/scielo.php?pid=S0034-

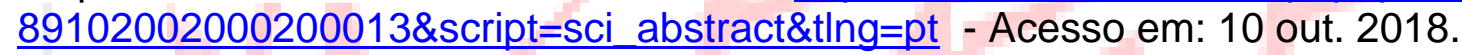

MANN, Luana. Alterações biomecânicas durante o período gestacional: uma revisão. Motriz, Rio Claro, 2010. Disponível em: https://s3.amazonaws.com/academia.edu.documents/43126546/Biomechanical_cha nges_during_pregnancy_a20160227-9379-

1700n51.pdf?AWSAccessKeyld=AKIAIWOWYYGZ2Y53UL3A\&Expires $=1553832440$ \&Signature $=3 \mathrm{hTV} 7 \mathrm{t} 5 \mathrm{cDXaBys}$ bog $6 \mathrm{nP} 90 \mathrm{KqPU} \% 3 \mathrm{D} \&$ response-contentdisposition=inline\%3B\%20filename\%3DAlteracoes_biomecanicas_durante_o_period. pdf . Acesso em: 30 set. 2018.

MANTELLI, Erica. Benefícios da musicoterapia na gestação para seu bebê. Blog bebê genial. Disponível em: https://bebegenial.com.br/2019/01/26/beneficios-damusicoterapia-na-gestacao/. Acesso em: 30 mai. 2019.

MATA, Junia; SHIMO, Antonieta. A representação social da arte da pintura do ventre materno para gestantes. Revista Pesquisa Qualitativa, São Paulo, 2017. Disponível em: https://editora.sepq.org.br/index.php/rpq/article/view/113/80. Acesso em: 26 set. 2018.

MONTENEGRO, Carlos Antonio Barbosa; REZENDO FILHO, Jorge de. Obstetrícia Fundamental. 14. ed. São Paulo: Guanabara, 2010 
MOREIRA, Thereza. Conflitos vivenciados pelas adolescentes com a descoberta da gravidez. Revista Esc Enfermagem USP, São Paulo, 2007. Disponível em: http://www.scielo.br/pdf/reeusp/v42n2/v42n2a14.pdf. Acesso em: 9 out. 2018.

PICCININI, Cesar. Gestação e a constituição da maternidade. Psicologia em Estudo, São $\quad$ Paulo, 2008.2 Disponível em: https://www.lume.ufrgs.br/handle/10183/98570. Acesso em: 4 out. 2018.

PICON, J. D. Alterações Hemodinâmicas da Gravidez. Revista da Sociedade de Cardiologia do Rio Grande do Sul. Ano XIV n.05.2005. Disponível em: http://sociedades.cardiol.br/sbc-rs/revista/2005/05/Artigo01.pdf. Acesso em: 30 mai. 2019.

SANTOS, Cesar. Terapia através da massagem:massoterapia no centro de atenção psicossocial de Amargosa/BA. Revista Acadêmica Gueto, São Paulo, 2015. Disponível

em: https://web.a.ebscohost.com/abstract?direct=true\&profile=ehost\&scope

$=$ site $\&$ authtype $=$ crawler $\&$ jrnl $=15189740 \& A N=134147907 \& \mathrm{~h}=5 \mathrm{E} 9$ UQR8IdBEWEnXsU v0ECzG\%2bBfmdtmDGwXuGJ\%2bz0XCiPY3h1nLte9be7bdg3jwjHfKn6WpmBH3owf TmLpxif0g\%3d\%3d\&crl=c\&resultNs=AdminWebAuth\&resultLocal=ErrCrINotAuth\&crl hashurl=login.aspx\%3fdirect\%3dtrue\%26profile\%3dehost\%26scope\%3dsite\%26auth type\%3dcrawler\%26jrnl\%3d15189740\%26AN\%3d134147907 Acesso em: 26 set. 2018.

SILVA, Nelyanna; MEJIA, Dayana. A utilização de recursos estéticos durante a gravidez. In: SILVA, Nelyanna; MEJIA, Dayana. A utilização de recursos estéticos durante a gravidez. 2014. Tese (Pós Graduação em Estética e Cosmética) Faculdade Sul Americana, São Paulo, 2012. Disponível em: http://portalbiocursos.com.br/ohs/data/docs/39/07 - A utilizaYYo de recursos estYticos_durante_a_gravidez.pdf. Acesso em: 27 nov. 2018.

URASAKI, Maristella. Cuidados com a pele adotados por gestantes assistidas em serviços públicos de saúde. ACTA, São Paulo, 2011. Disponível em: http://www.scielo.br/pdf/ape/v24n1/v24n1a10.pdf. Acesso em: 26 set. 2018. 